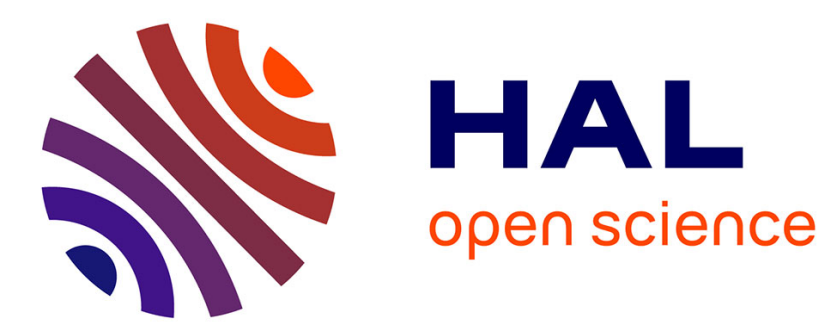

\title{
Les épées à sphères du Cayla à Mailhac (Aude)
}

Odette Taffanel, Jean Taffanel

\section{To cite this version:}

Odette Taffanel, Jean Taffanel. Les épées à sphères du Cayla à Mailhac (Aude). Gallia - Fouilles et monuments archéologiques en France métropolitaine, 1967, 25 (1), pp.1-10. 10.3406/galia.1967.2461. hal-01934399

\section{HAL Id: hal-01934399 \\ https://hal.science/hal-01934399}

Submitted on 4 Feb 2020

HAL is a multi-disciplinary open access archive for the deposit and dissemination of scientific research documents, whether they are published or not. The documents may come from teaching and research institutions in France or abroad, or from public or private research centers.
L'archive ouverte pluridisciplinaire HAL, est destinée au dépôt et à la diffusion de documents scientifiques de niveau recherche, publiés ou non, émanant des établissements d'enseignement et de recherche français ou étrangers, des laboratoires publics ou privés.

\section{(이) $\$$}

Distributed under a Creative Commons Attribution - NonCommercial - NoDerivatives| 4.0 


\title{
LES ÉPÉES A SPHÈRES DU CAYLA A MAILHAC
}

\section{( $A \cup D E)$}

\author{
par O. ef J. TAFFANEL
}

Les campagnes de fouilles de 1965 et 1966 ont été consacrées à l'exploration d'un fossé doublant le rempart de la quatrième ville du Cayla ${ }^{1}$ à mi-pente de la colline (fig. 1 et 2). Nous l'avons vidé sur $25 \mathrm{~m}$. 50 de ìng. C'est un fossé de section triangulaire, creusé dans des marnes stériles rouges, jaunes ou bleues, surmontées dans une partie de la paroi sud par un banc de grès tendre (fig. 3). Le profil actuel, qui est celui du fossé au moment $\mathrm{du}$ comblement, révèle le travail des eaux, qui ont principalement afiouillé la paroi sud audessous du banc de rocher (fig. 4 et $5, n^{0} 2$ ). A 19 mètres, le banc de rocher qui préservait la marne disparait et la paroi sud a complètement cédé à cet endroit (fig. 3). Comme la pente nord présentait en face d'importants glissements, on a l'impression qu'il y a eu, à la suite de fortes pluies, une sorte de cascade qui a crevé le fossé à cet endroit. Cependant, on peut aisément rétablir la section régulière du fossé au moment du creusement (fig. 4 , en pointillé), grâce à l'érosion qui a conservé dans le sol dur le tracé du fond primitif.

La partie de la paroi nord comprise entre le $14 \mathrm{e}$ et le $22^{\mathrm{e}}$ mètre était creusée dans un terrain déjà archéologique dans la partie supérieure, où la marne irrégulière avait retenu dans les creux d'importants dépòts de Cayla I pur ${ }^{3}$, surmontés d'apports moins nets Cayla II et III. Ces observations nous font penser que le fossé a été creusé au début du Cayla IV et qu'il est en relation directe avec le rempart de cette époque. Cela expliquerait très bien son

(1) Sur la stratigraphie du Cayla, voir surtout M. Lot s, O. et J. TAFraxis, Le premier age du Fer languedocien,

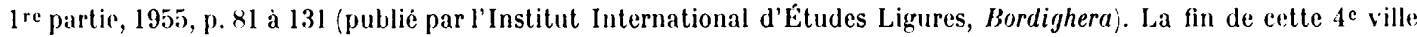
est assez facile a préciser, car les traces d'un incendie total y sont nettes. Par contre, le début en est plus imprécis, car la ville précédente, la $3^{\mathbf{c}}$, n’est que partiellement incendiée et une bonne partie de son matériel céramique importé est passé dans la quatrième. On ne peut donc ici se fier aux vases importés les plus anciens pour dater le début de la quatrième ville. Il faudrait fouiller davantage la partie incendiée de la troisième ville pour préciser la date des importations les plus tardives qu'elle renferme, et aussi découvrir les tombes correspondant a ces deux villes. Actucllement, les dates qu'on peut proposer pour les quatre villes du Cayla sont: I, Ixe-vII's.; II, 600-475; III, 475)-300) (?); IV, $300(?)-75$.

(2) Le profil d'ensemble correspondrait a celui du fossé de Vix, avec cependant des dimensions moindres (1R. Joffroy, L'oppidum de Vix el la civilisation hallstattienne dans l'Est de la France, 1960, p. 19 et pl. 4, fig. 1).

(3) Cette première ville du Cayla ne renferme encore aucune céramique importée. Nous avons fouillé dans la plaine 300 tombes à incinération correspondant à cette ville (nécropole du Moulin), dont le mobilier complète celui des habitations. En gros, on peut dater cette période des $\mathrm{Ix}^{\mathrm{e}-\mathrm{v} \mathrm{II}^{\mathrm{e}}}$ s. avant J.-C. (voir Le premier âge du Fer languedocien, $2^{e}$ partie, 1958, p. 7-30). 


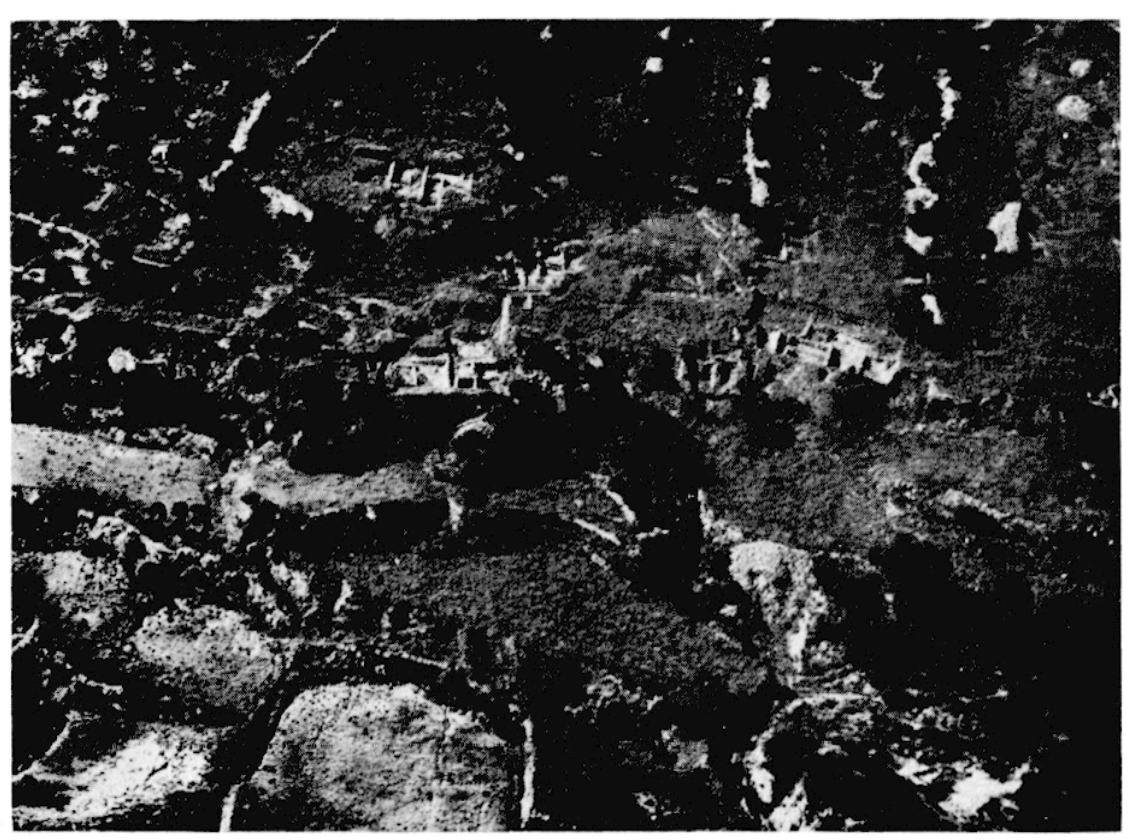

1 Vue aérienne de la colline du Cayla avant la fouille du fosse (an-dessous de la fouille 17, daus le champ no $: 2: 3^{3}$.

aspect dégradé, car l'herbe pousse difficilement dans cette marne et il aurait eu environ deux siècles d'existence au moment du comblement. le fossé a été comblé d'une seule venue. avec une terre renfermant d'innombrables vestiges, tessons, objets en métal et en pierre, déchets de nourriture, qui appartiennent aux quatre premiers villages du Cayla et proviennent manifestement des déchets accumulés au cours des siècles au pied du rempart.

Les vestiges les plus récents trouvés dans ce remplissage placent dans le courant du I $^{\text {er }}$ siècle avant J.-C. le comblement du fossé. Nous y avons remarqué trois éboulis, qui correspondent à la démolition du rempart :

10 tout au fond (surtout de grosses pierres).

20 à mi-hauteur (fig. $5, \mathrm{n}^{0} \overline{5}$ ),

$3^{\circ}$ tout à la surface du comblement (fig. 5, nos 3-4).

Mais à partir du dix-septième mètre un élément insolite apparut à mi-hauteur du remplissage. Sensiblement au même niveau, des objets en fer étaient d'abord éparpillés, puis atteignaient une forte densité entre le douzième at le septième mètre, où ils formaient un véritable tas de ferraille, et s'éparpillaient à noureau vers la paroi sud, jusqu'à la limitr actuelle de la fouille. La tres forte oxydation de ces objets ne permet pas toujours de less identifier avec certitude, et il convient d'attendre leur restauration pour less étudier en détail4. Cependant, nous y avons parfaitement reconnu 16 e epées à sphères, dont 14 poignées entières (nos 9, 11, 15, 16, 28, 30, 34, 38, 41, 65, 66, 69, 71, 96, fig. 6, 7 et 8), 1 dont le "rognon " manque ( $\mathrm{n}^{0} 39$, fig. 7 ) et 1 incomplete, avec une seule sphère de la garde $\left(\mathrm{n}^{0} 42\right.$, fig. 7).

(4) M. J. Bouis, Ingénieur chargé de la restauration des objets de fonilles ì Marseille, s'occupe de ce travail et de l'étude des techniques employées pour forger ces épées. 


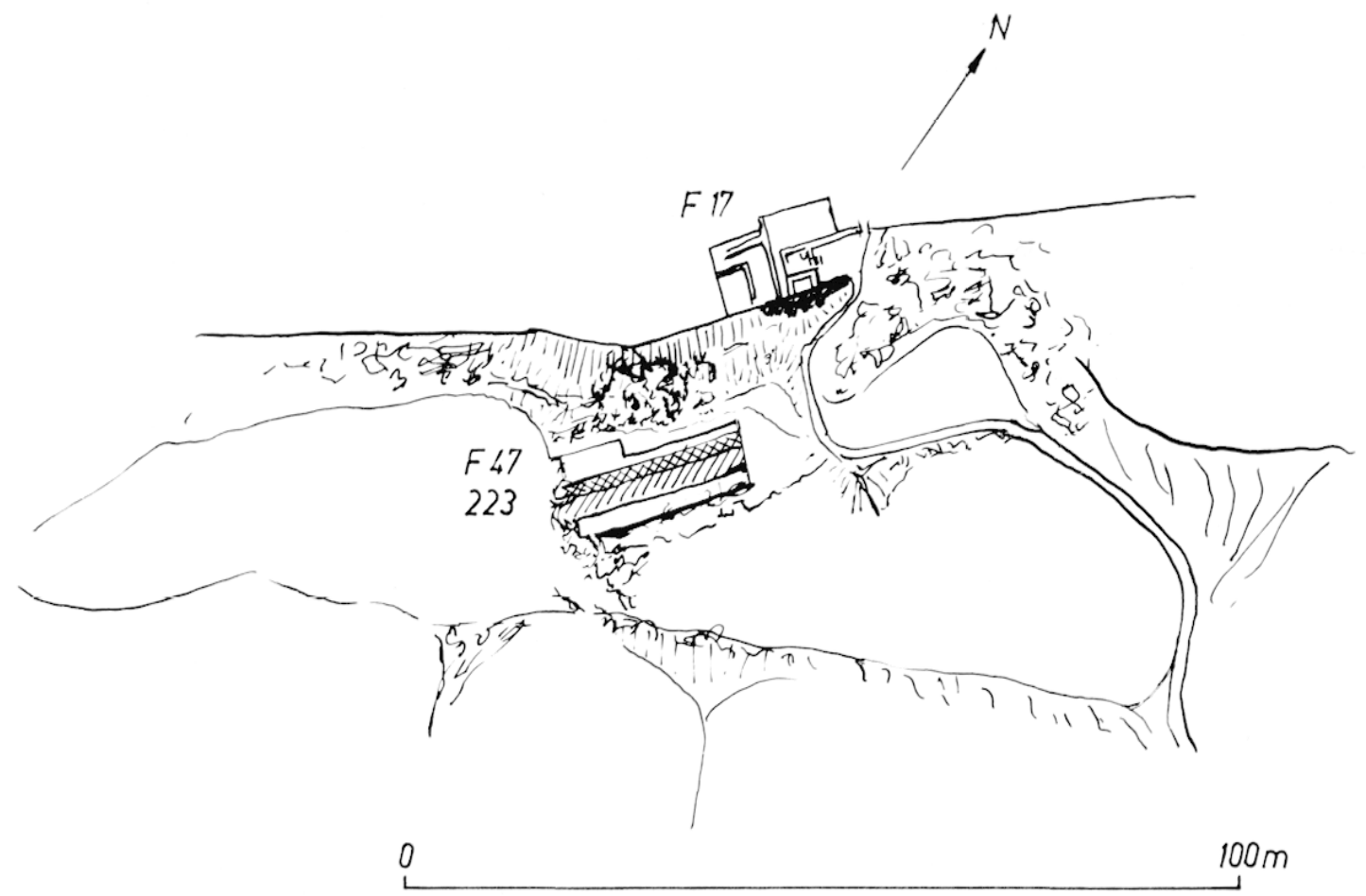

2 I.e Ciayla. Fossé (fouille 47 ,

T'outes appartiennent au type 1 de W. Krämer ${ }^{5}$ mais aver, semble-t-il, des variantes dans la longueur et la largeur des lames. Cependant, il y a aussi dans notre dépòt des fragments de lames fines et étroites pareilles a celles du type 1. Toutes, sans exception, étaient dans leur fourreau de fer plus ou moins bien conservé, toutes sont cassées ou torlues. Six d'entre elles ont encore leur dispositif de suspension fixé au fourreau (n ${ }^{0 s} 15,16,30$, 65), 66, 69, fig. 7). Il faut y ajouter la mème pièce de suspension fixée au fourreau du fragment no 46 et probablement une autre isolée (no 62 ), soit 8 au total.

Rappelons-le, jusqu'i cette découverte on ronnaissait seulement 12 épres de ce type $1^{6}$ et aucune dans le Midi de la France? I' 'autre part, les essais de datation de cette arme étaient tous fondés sur des observations typologiques, car les 3 trouvées en fouilles 8 n'avaient fait l'objet d'aucune observation archéologique précise, et toutes les autres ont

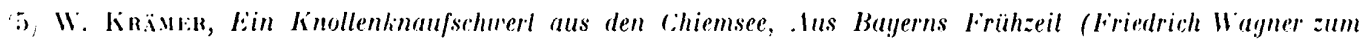
i.j (ieburtslag), 1960, 1. 110, fig. 1, n" I. A lheure acturlle, cest l'etude la plus complite sur cette question, oi l'auteur donne tous les renseignements connus sur ces riperes, toutes réunies dans sa fig. 2. L'étude radiographirfur, trés poussie, n'a pu être réalisée que sur l'épée du chiemsere, mais elle révele des particularities de fabrication triss curienses.

6) Le type 2 en differe sensiblement par la disposition des yuatre spheres du haut, placies sur une ligne a peine cintrée, ce qui entraine un èlargissement du corps de la poigncée.

(7) En voici la repartition : 4 en Allemagne (Chiemser, L.Im, Eislingen, Lengenfeld), 1 en suisse, 7 'n liraner :

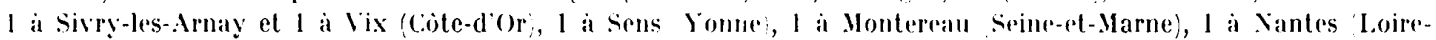
Atlantique; et $:$ douteuses dans les Deux-Sivres.

(8) Celle de "L.engenfeld *, dont le lieu mème de trouvaille n est pas exactement situé, celle de sirry-les-Arnay, trouvée par un paysan, dans un tumulus arasé, semble-t-il, avec des objets de l'àge du Bromze, ct celle de Sens. 




3 Ouadrillage de la fouille et emplacement des ipées.
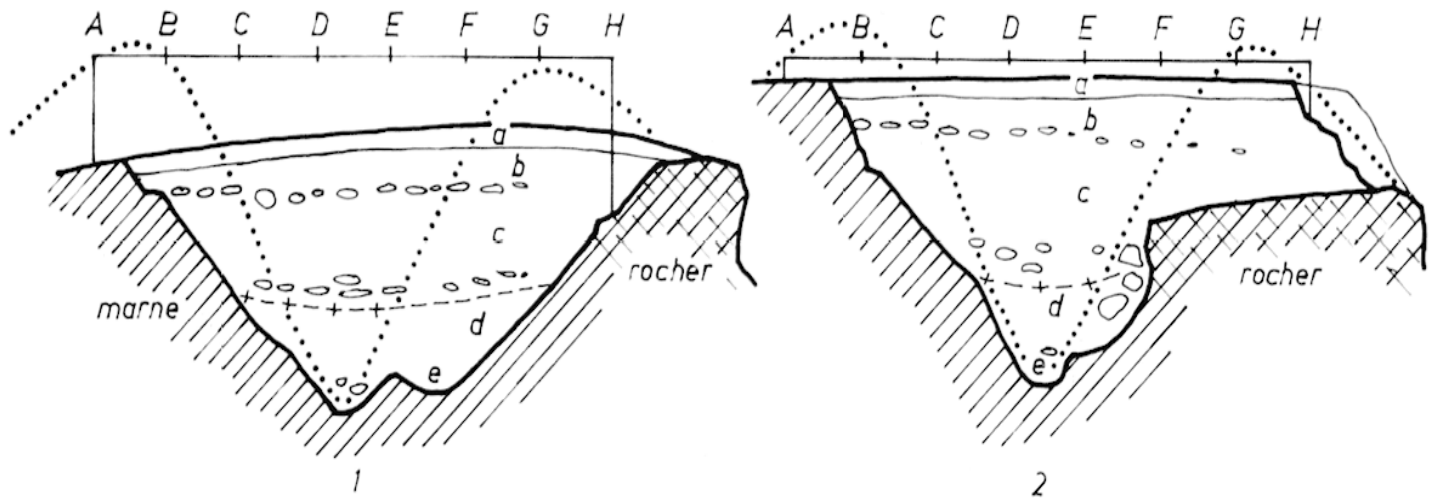

4 1) Section du fossé à 9 mètres. 2) Section à 1 mètre. Lin pointillí, section probable du fossé primitif. niveau du depót de fer.

dete pèchées dans des lleuves ou des lars. Notre fouille en étend l'aire de répartition et permet de circonscrire le problème archéologiqur.

Les apports postérieurs au comblement du fossé. sur l'éboulis supérieur, niveaux a et $b$ (a étant la partie supérieure du niveau remaniée par les labours). les seuls normalement stratifiés, nous ont en effet donné des fragments d'Arezzo et de la Ciraufesenque, ces derniers très abondants. Par contre, l'abondant maleriel céramique du comblement n'en renfermait pas la moindre parcelle. I'où une première certitude : le comblement du fossí est antérieur à l'apparition de l'Arezzo dans nos régions, c'est-à-dire à l'époque d'Auguste.

Au-dessous, dans la zone du dépòt de fer, nous avons subdivisé le comblement en niveau $c$, au-dessus du dépôt, $d$, au-dessous du dépôt, et $e$, tout au fond, dans les creux 

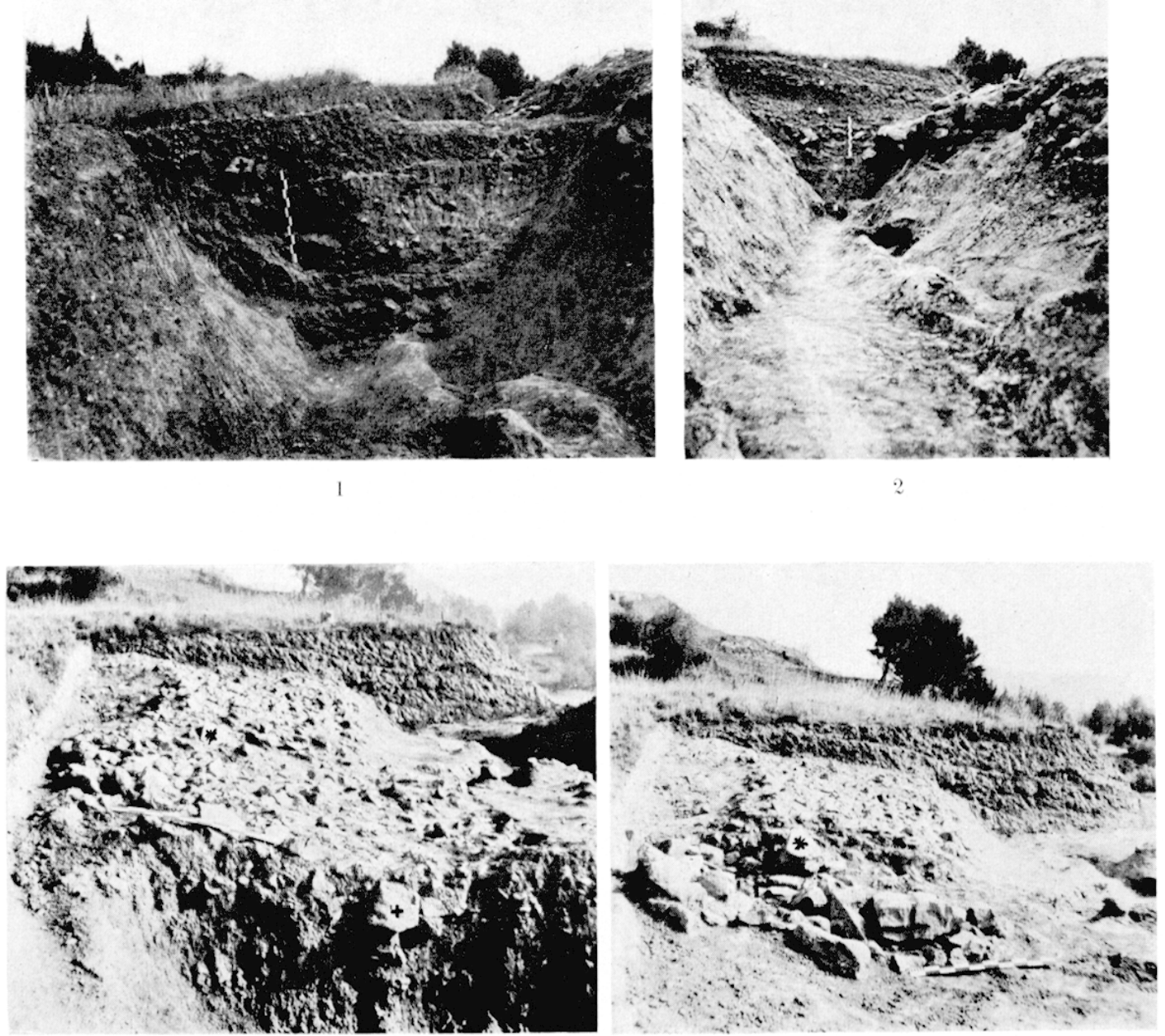

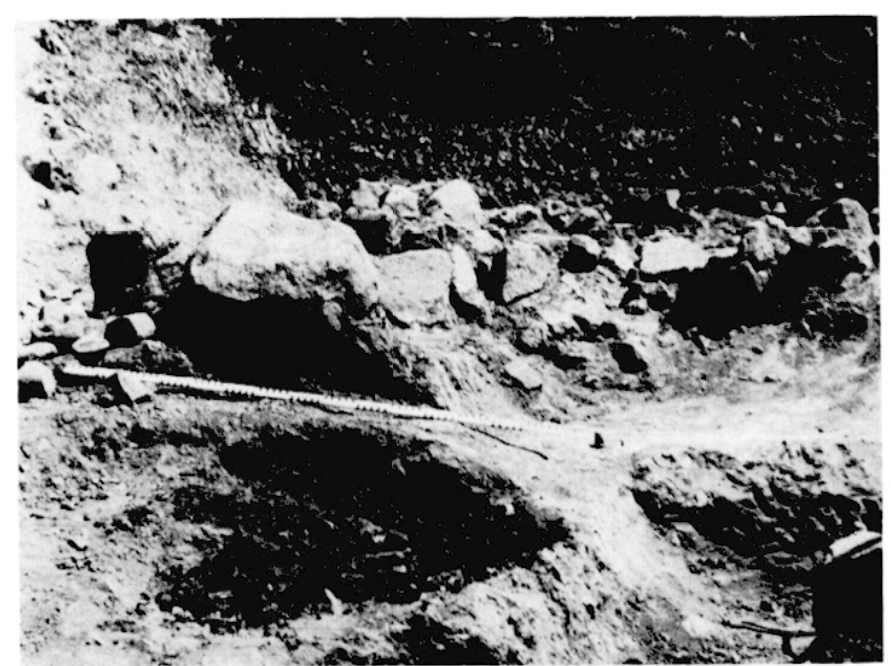

5
5) 1 Section du fosse a 9 mitres. 2) Siection i I metre. Sur ces deux photos, le bas de la reggle indique le nivean du dépót de fer. 3) Surface de l'oboulis supérieur, de 1 i 9 m. L Structure du même éboulis montrant les grossers pierres noyiers dans la pierraille. 5) Deuxieme éboulis, juste audessus du depoit de fer indiqué par la règle horizontale 


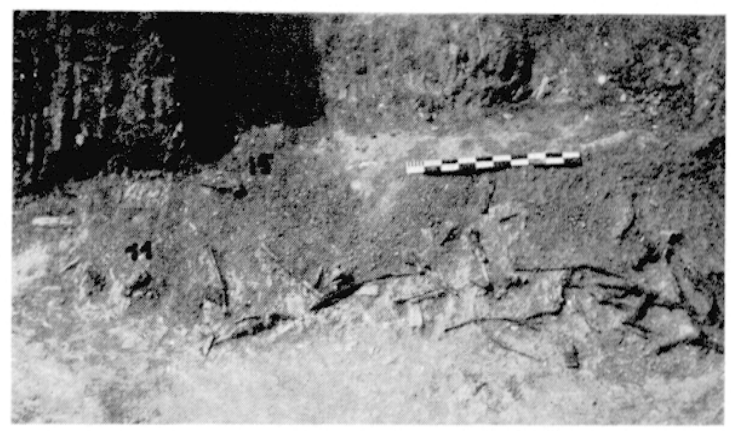

1

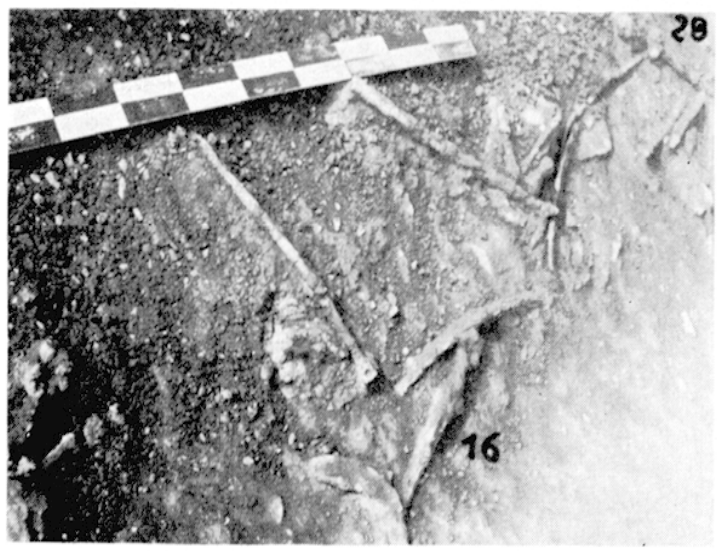

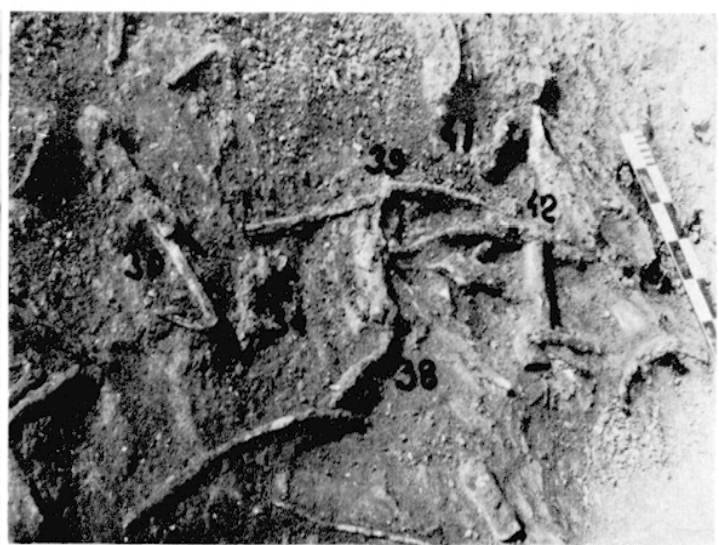
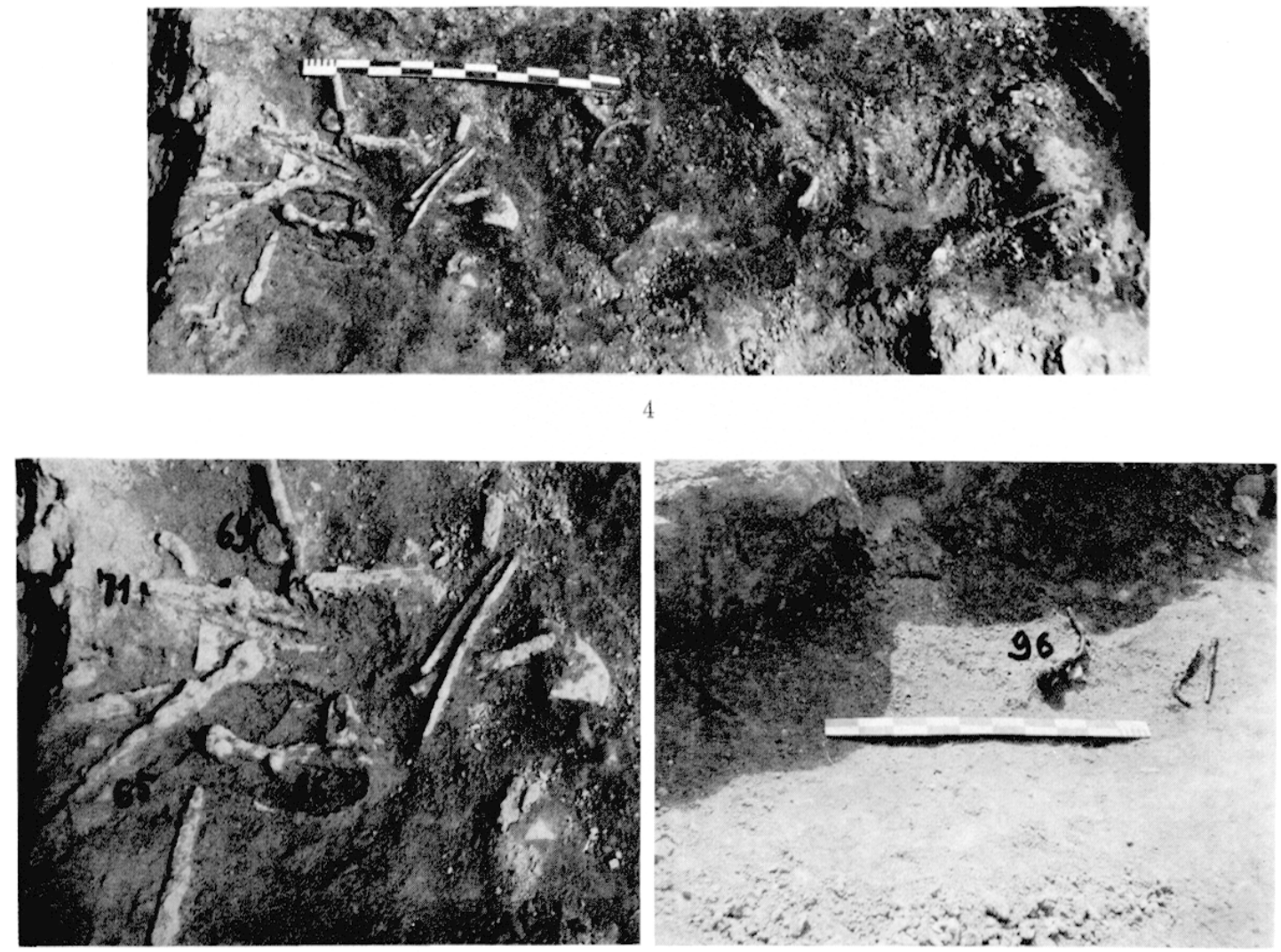

6 1) Partie du dépơl décapéc en 1965. 2 el 3) Détails monlranl les épées mêlées aux autres objets. 4) Suite du dépôt, décapée en 1966.5 et 6 ) Détails montrant les épées en place. 

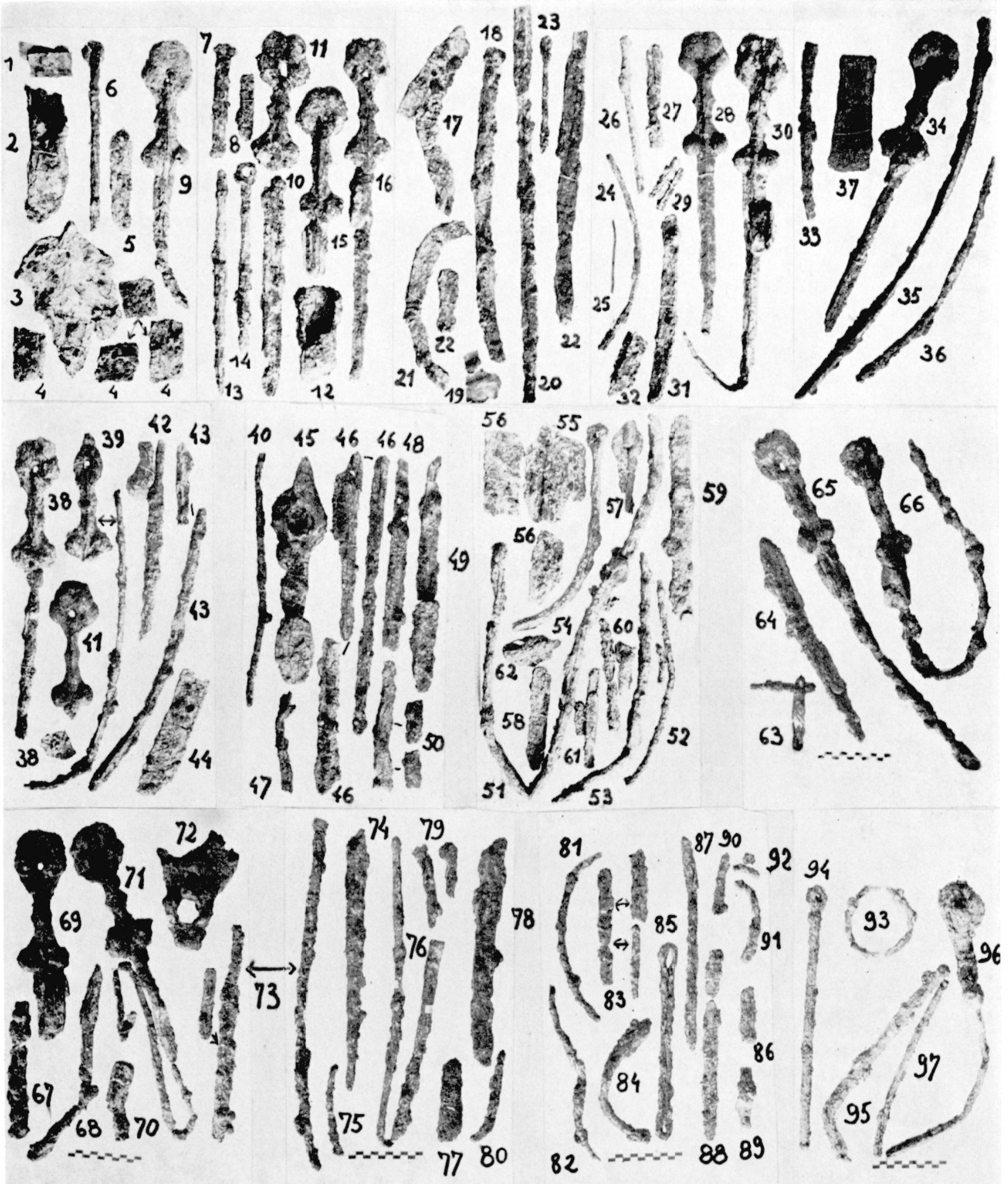
(fig. 4). Chaque tesson portant ces indications stratigraphiques, nous nous sommes aperçus, au remontage, que des fragments de mêmes vases, qui se raccordent, provenaient à la fois du $c$ et du $d$ (fig. $9, n^{08} 2,3,4$ ). C'est la preuve formelle que le remplissage est bien homogène : le niveau marqué par le dépòt de fer correspond seulement à une brève interruption, le temps d'y jeter ces objets, puis le comblement a été repris avec les mêmes détritus. Au-dessous du dépôt, dans le $d$, un fragment de coupe campanienne de type B, forme $1 \mathrm{~A}$ (fig. $9, \mathrm{n}^{\circ} 1$ ), nous donne une date plus précise, puisque $\mathrm{N}$. Lamboglia place son apparition au I ${ }^{\text {er }}$ siècle avant J.-C. ${ }^{9}$. On peut donc penser que le fossé a été comblé dans la première partie du I $^{\text {er }}$ siècle avant J.-C.

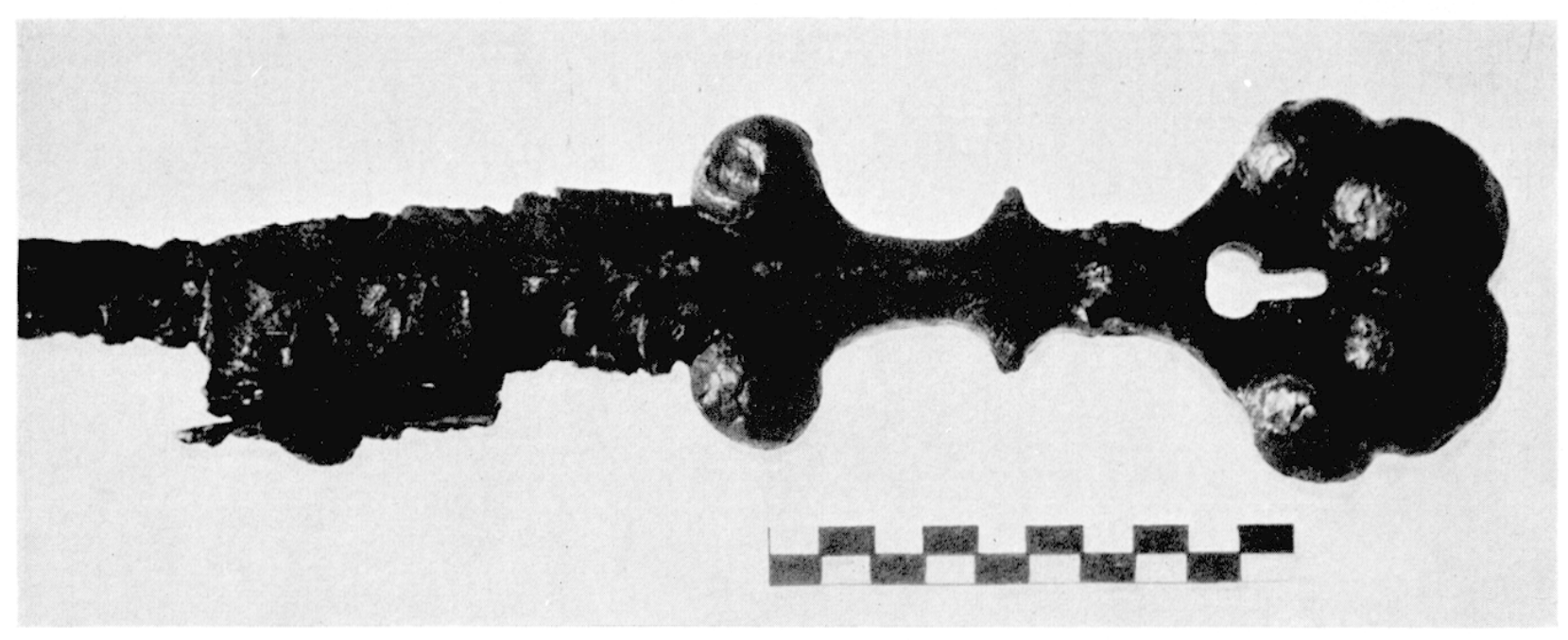

8 Épée no 30 après restauration.

Cie comblement est en corrélation avec le démantèlement de la quatrième ville, qui est incendiée. Incendie total de la ville, démolition systématique du rempart, comblement du fossé ainsi que du puits $\mathrm{n}^{0} 39$, situé dans la villẹ: ${ }^{10}$, autant d'indices qui font penser à des mesures de police destinées a réduire à l'impuissance la population du Cayla ${ }^{11}$. La quatrième ville est une ville celtisée, où l'on employait l'alphabet ibère : on y trouve à la fois des vases "gaulois", des monnaies à la croix des Tectosages, des monnaies ibères de Narbonne et des graffites ibères sur des tessons. Or, à l'époque indiquée par la céramique la plus récente du fossé, du puits et des maisons incendiées, se place, en 76-74 av. J.-C., le gouvernement de II. Fontéius, qui fut précisément marqué par d'impitoyahles répressions. In passage du Pro Fonleio semble s'appliquer tout particulièrement à une révolte des tribus voisines de Narbonne: "Parmi les défenseurs de M. Fontéius, on trouve encore la colonie

(9) Nino Lambogins, Per unu classificazione preliminare della ceramica campana, Actes du ler Congrès International d'Études ligures, 1952, p. 143-144.

(10) Voir Le premier âge du Fer languedocien, $1^{\text {re }}$ partie, p. 84 et fig. 55. L.e mobilier trouvé dans ce puits est encore inédit.

(11) Nous avions d'abord pensé que c'était au moment où les Komains ont fondé la colonie de Narbonne, mais une meilleure datation des poteries donne une époque plus tardive. 


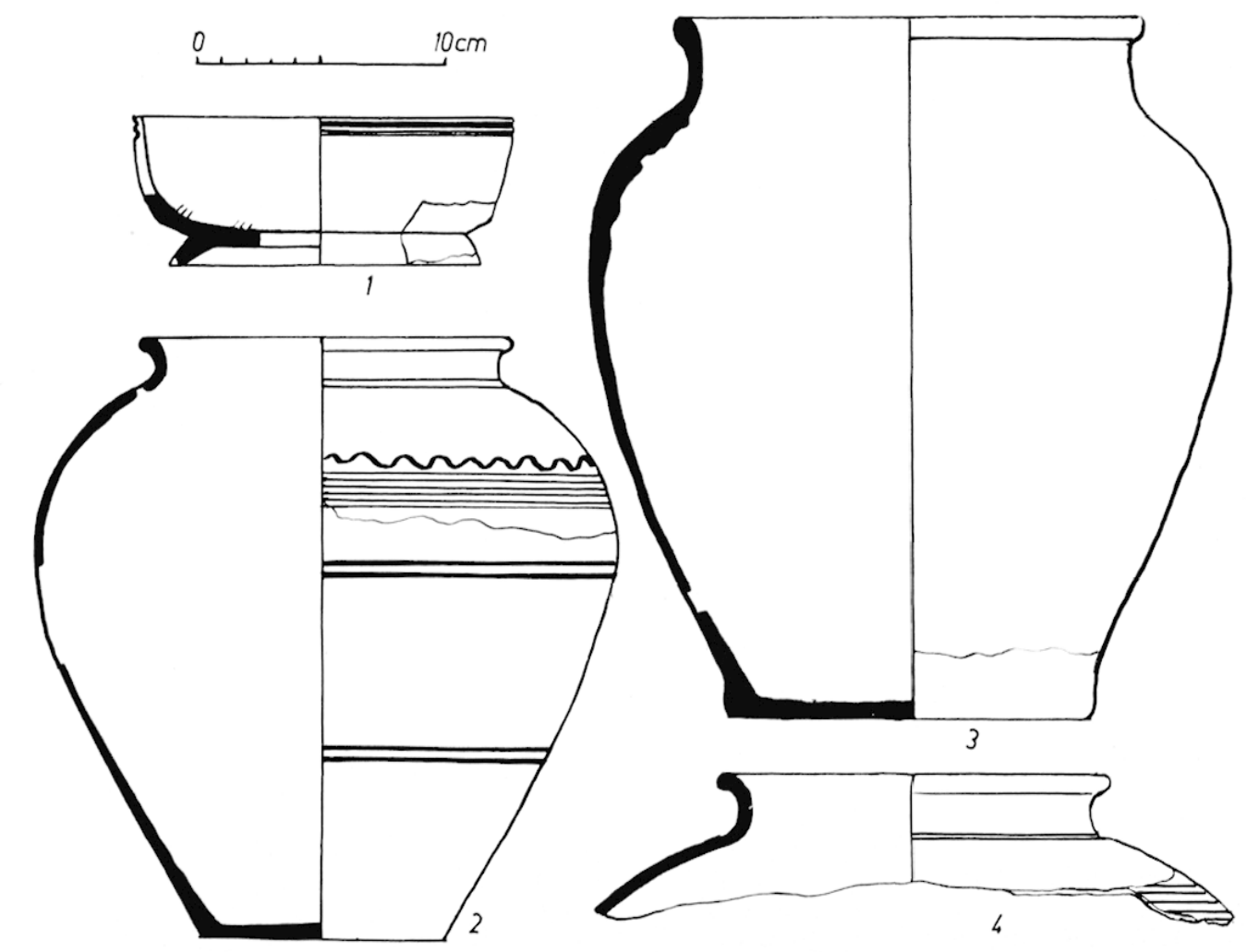

9 1) Fragment de coupe campanienne trouvé dans le niveau $d$ (au-dessous des épées). 2, 3 et 4) Vases dont les fragments étaient éparpillés au-dessus et au-dessous des épées ( $c$ et $d$ ). 2 et 4 ) Poterie gris-clair fine, décor lissé, par dessus un engobe plus clair dans la partie supérieure. 3) Poterie gris-clair dure el rugueuse, pellicule noire en surface, sauf sur un point où elle est du même gris-clair qu'à l'intérieur, lissée en dehors jusqu'à $3 \mathrm{~cm}$ du fond.

de Narbonne; récemment délivrée par lui des ennemis qui la menaçaient de près, elle se montre aujourd'hui touchée de son infortune et de ses périls $\|^{12}$.

Toutefois, même si notre hypothèse est exacte, elle ne nous donne aucune explication sur la nature mème du dépòt de fer enseveli dans le fossé. On peut, croyons-nous, écarter l'hypothèse de ferrailles déjà jetées au rebut et mêlées aux déchets qui ont servi à remplir lo fossé, car dans ce cas nous les aurions trouvées disséminées, comme les tessons, dans toute l'épaisseur du comblement. Même en admettant qu'on ait pu mettre à part les épées, nous ne voyons pas pour quelle raison on aurait trié aussi minutieusement tous les petits fragments. Ce dépôt forme un tout, mais se compose surtout d'objets cassés, tordus ou repliés, à l'exception de petites barres épaisses, de dimensions assez uniformes ( $\mathrm{n}^{\text {os }} 10,49$, $58,64,78,82$ etc.), qui paraissent être des saumons de fer brut. Une seule hypothèse expliquerait ces particularités : celle d'un fonds de forge, où l'artisan avait réuni, à côté du fer non encore façonné, des objets hors d'usage destinés à être reforgés. Cette forge aurait très bien pu se trouver à l'aplomb du dépôt entre le fossé et le rempart, dans la zone encore inexplorée. S'il en reste des traces, la fouille nous les révèlera. Dans ce cas, les épées à sphères

(12) CicÉron, Pro Fonteio, XX, 46 : ‘ Propugnal pariler pro salule M. Fontei Narbonensis colonia, quae per hunc ipsa nuper obsidinne hostium liberala, nunc eiusdem miseriis ac periculis commovetur... ". 
auraient été détruites au début du rer siècle avant J.-C. Ciela ne nous dit pas à quel moment clles ont été forgées mais, comme il y a uniquement ce type d'armes dans ce dépôt, nous ne les croyons pas antérieures de plusieurs siècles à cette date. Bien qu'on ait jusqu'ici communément placé ces épées a l'époque hallstattienne, W. Krämer envisage cependant dans son itude précitér leur attribution possible à La l'ène moyenne ou finale'3.

Les tombes de guerriers de la fin du II $^{\mathrm{e}}$ et du Ir $^{\mathrm{e}}$ siècle avant .J.-(C. sont encore pratiquement inconnues dans notre région. Par contre, les tombes du vie siècle avant J.-C. de Mailhac ${ }^{14}$ et de Pézenas ${ }^{15}$ ont donné des épées et des poignards à antennes classiques, et les tombes d'Ensérune, dont les plus récentes sont datées de - 225 environ ${ }^{16}$, de non moins rlassiques épées de Ia Tène I et II. Seule, l'étude approfondie des techniques de fabrication de nos épées à sphères, à laquelle travaille II. Bouis, nous permettra de savoir si elles sortent des mêmes ateliers que les épées de ce type déjà connues ${ }^{17}$. Bien d'autres problèmes s'y rattachent, que nous étudierons dans une prochaine publication, quand les objets seront nettores et irlentifiés.

Odelte et Jean TAFfanes.

(13) W. KR̈̈MI:R, op. cil., p. 118.

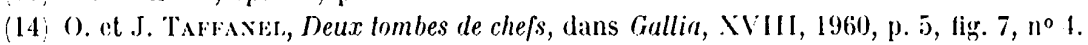

(15) Abbé J. Giry, Nècropole de St-Julien, dans Échos et Documents, mai 1966, p. 10.

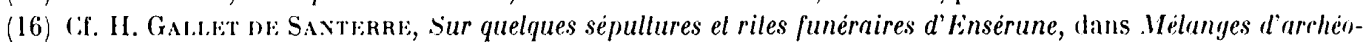
logie el d'histoire offerts à André Piganiol, 1966, p. 1027.

(17) Dans notre région, une épée pourrait être considérée comme le prototype des épées à sphères de type 1. Elle provient de la nécropole St-Julien de P’ézenas 'Abbé Grry, op. cit., p. 7) mais, malheurensement, elle a été remontée par le labour et rien ne permet de la dater exactement. 